\title{
Synthesis of an IS and Steviol Glycoside Analysis by a Validated Internal Standard Method
}

\author{
Jan M. C. Geuns ${ }^{1}$, Tom Struyf ${ }^{1}$, Stijn Ceunen ${ }^{1}$, Gert Steurs², Wim De Borggraeve ${ }^{2}$ \\ ${ }^{1}$ Laboratory of Functional Biology, KU Leuven, Leuven, Belgium \\ ${ }^{2}$ Division of Molecular Design and Synthesis, KU Leuven, Leuven, Belgium \\ Email: Jan.Geuns@bio.kuleuven.be
}

How to cite this paper: Geuns, J.M.C., Struyf, T., Ceunen, S., Steurs, G. and De Borggraeve W. (2018) Synthesis of an IS and Steviol Glycoside Analysis by a Validated Internal Standard Method. American Journal of Analytical Chemistry, 9, 547-559.

https://doi.org/10.4236/ajac.2018.911040

Received: September 1, 2018

Accepted: November 4, 2018

Published: November 7, 2018

Copyright $\odot 2018$ by authors and Scientific Research Publishing Inc. This work is licensed under the Creative Commons Attribution International License (CC BY 4.0).

http://creativecommons.org/licenses/by/4.0/

(c) (i) Open Access

\begin{abstract}
The internal standard (IS) method is the best method for the analysis of samples, as it is independent of errors in injection volume, changes in sample volumes, and changes in sensitivity of the detector, etc. Use of an internal standard allows for the correction of losses due to sample clean-up of complex samples. An ideal IS is a compound that has properties very similar to, and that behaves as the compounds to be analysed. Ideally, only in the last step of analysis (HPLC), the IS should be well separated from the compounds of the mixture to be analysed. After testing several existing compounds with
\end{abstract} negative results, we decided to synthesise the

$19-O-\beta$-D-galactopyranosyl-13- $O-\beta$-D-glucopyranosyl-steviol as IS. This is the 19-galactosyl ester of steviolmonoside (13-O- $\beta$-D-glucopyranosyl-steviol). The IS was made according to published methods. Steviolmonoside (SM) was made from purified commercial rubusoside (Rub) by refluxing it in $10 \%$ $\mathrm{KOH}$ for $2 \mathrm{~h}$. SM was precipitated and crystallized from $\mathrm{MeOH}$. The hydroxyls of the glucose unit of SM were protected by acetylation. The acetylated SM was crystallized from acetone and dissolved in 1,2-dichloroethane. Then $\mathrm{Ag}_{2} \mathrm{CO}_{3}$ on Celite and tetra-acetylated galactopyranosyl bromide were added and the mixture was refluxed for $2 \mathrm{~h}$. After cooling, $\mathrm{BaO}$ in $\mathrm{MeOH}$ was added to remove the acetyl groups. The 1,2-dichloroethane fraction was then extracted three times with equal volumes of water and the water fraction containing the IS was further purified on a $\mathrm{C}_{18}$ flash chromatography column. Traces of unreacted SM were removed by preparative HPLC on an Alltima $\mathrm{C}_{18}$ column $(250 \mathrm{~mm} \times 22 \mathrm{~mm}$, particle size $10 \mu \mathrm{m})$ with AcCN:water (35:65, $20 \mathrm{ml} / \mathrm{min}$ ). Detection was at $210 \mathrm{~nm}$ (KNAUER, "Smartline" UV detector 2500). The collected IS fraction from the HPLC was completely dried. Mixtures of steviol glycosides (SVglys) containing IS could be purified over SPE 
cartridges without change of the SVgly over IS ratio. The calibration curves for rebaudioside A (RebA) and stevioside (ST) were linear between 0.012 and 0.95 and between 0.013 and $1.13 \mathrm{mM}$ for RebA and ST, respectively. The accuracy was checked by the standard addition method. It was concluded that the IS method gives an excellent precision and accuracy.

\section{Keywords}

Steviol Glycosides, Internal Standard Method, Validation, Quantification, Purification

\section{Introduction}

For many years, steviol glycosides (SVglys), the sweet diterpene glycosides found in Stevia rebaudiana Bertoni leaves, have been widely used as intense sweeteners. In several countries their use is allowed in general food (China, Brazil, India, Japan, ...) or as a food additive (Australia, New Zealand, USA, EU). The Joint FAO/WHO Expert Committee on Food Additives (JECFA) as well as EFSA proposed an Acceptable Daily Intake (ADI) of $0-4 \mathrm{mg}$ steviol equivalents $/ \mathrm{kg}$ BW. The required purity of the mixture is 95\% [1] [2] [3] [4] [5].

The analysis of SVglys is usually done with HPLC using $\mathrm{NH}_{2}, \mathrm{C}_{18}$, carbohydrate or HILIC columns [6] [7] [8] [9]. Although $\mathrm{NH}_{2}$ columns give a good separation, they have poor reproducibility and are not practical [7]. $\mathrm{C}_{18}$ columns are more robust but may give poor resolution. This can be solved by using two columns in series [10]. Recently, there is a shift from using $\mathrm{NH}_{2}$ columns to using $\mathrm{C}_{18}$ columns. Moreover, JECFA recommended the use of a $\mathrm{C}_{18}$ column [3].

The detection of SVglys is usually done with an UV-detector, an evaporative light scattering detector (ELSD) or a mass spectrometer [6] [8] [9] [11] [12] [13] [14].

For the time being, all quantitative analyses of SVglys have been done with an external standard method. In many cases, rebaudioside A (RebA) is used as external standard and as a reference to quantify all occurring SVglys. This can be done if calibration curves are made in $\mathrm{mM}$ concentrations as the extinction coefficients are very similar [7]. Although many suggestions were made to improve SVgly analysis by an external standard method, round-robin testings organised by EUSTAS showed that the RSD was still around 4.5\%. Only a few laboratories managed to obtain an analysis at $\pm 1.5 \%$ accuracy by taking into account all the given suggestions. However, the success of an analysis by an external standard is dependent upon so many factors that a perfect analysis remains very difficult to obtain. As the purity requirement is $95 \%$, a RSD of $4.5 \%$ by an external standard method cannot be accepted as the purity found might then fluctuate between $90.5 \%$ and $99.5 \%$ [9].

Since SVglys are used in different foods and beverages, it is preferable to use a validated internal standard method to quantify SVglys in these matrices. Such a 
method is independent of errors in injection volume, changes in sample volume and changes in sensitivity of the detector and all the factors described before [9]. The use of an internal standard also allows for the correction of losses due to sample clean-up of complex samples.

An ideal IS is a compound with chemical and physical properties very similar to the compounds to be analysed. Ideally, only in the last step of analysis (HPLC), the IS should be well separated from the compounds of the mixture to be analysed. After testing several existing compounds with negative results, we decided to synthesize the

$19-O-\beta$-D-galactopyranosyl-13- $O-\beta$-D-glucopyranosyl-steviol as an IS. This is the 19-galactosyl ester of SM (13-O- $\beta$-D-glucopyranosyl-steviol). Figure 1 shows the structure of this IS. This compound was chosen as an IS because it does not naturally occur in plant extracts [14] and it is well separated from known SVglys.

\section{Methods and Material}

\subsection{Solvents and Products}

Solvents and water used were of HPLC quality. Other products were of PA grade. Standards were crystallised to $>99 \%$ purity [15].

\subsection{Synthesis and Purification of IS}

The IS was made according to [16]. To prepare the IS, Rub was purified from a commercial mixture containing $70 \%$ Rub. Then SM was made by refluxing Rub in $10 \% \mathrm{KOH}$ for $2 \mathrm{~h}$. After acidification with acetic acid (100\%) to $\mathrm{pH} 5$, the SM was precipitated by placing the mixture in a freezer at $-20^{\circ} \mathrm{C}$. The precipitate was dissolved in warm $\mathrm{MeOH}$ and crystallized again. In the next step, the hydroxyls of the remaining glucose unit were protected by acetylation with acetic anhydride in pyridine $(1: 1)$ for $25 \mathrm{~h}$ at $37^{\circ} \mathrm{C}$ while shaking. After acetylation, water was added to the reaction mixture as well as acetic acid to obtain a $\mathrm{pH}$ of 4. The water fraction was extracted with diethyl ether. The ether phase was

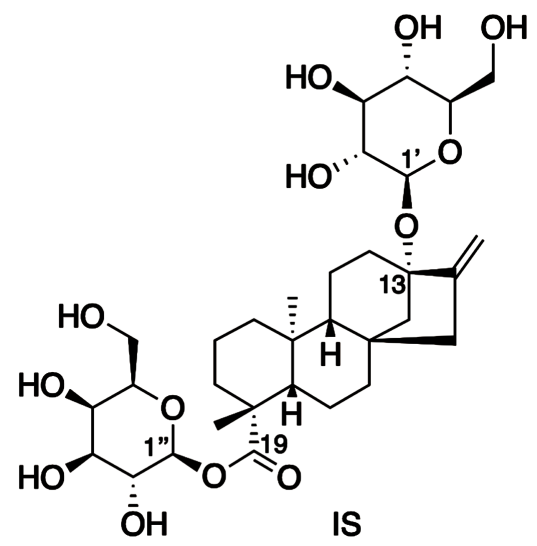

Figure 1. Structure of the IS

(19-O- $\beta$-D-galactopyranosyl-13- $O$ - $\beta$-D-glucopyranosyl-steviol). 
dried, and acetylated SM was crystallized from acetone. The acetylated SM was dissolved in 1,2-dichloroethane. Then $\mathrm{Ag}_{2} \mathrm{CO}_{3}$ on Celite and tetra-acetylated galactopyranosyl bromide were added and the mixture was refluxed for $2 \mathrm{~h}$. After cooling, $\mathrm{BaO}$ in $\mathrm{MeOH}$ was added to remove the acetyl groups. The 1,2-dichloroethane fraction was then extracted three times with equal volumes of water and the water fraction containing the IS was further purified on a $\mathrm{C}_{18}$ flash chromatography column. The column was rinsed with $20 \%$ acetonitrile in water and IS eluted with acetonitrile. The solvent was evaporated under reduced pressure at $50^{\circ} \mathrm{C}$. Because the IS still contained traces of unreacted SM, further purification by preparative HPLC on an Alltima $\mathrm{C}_{18}$ column $(250 \mathrm{~mm} \times 22 \mathrm{~mm}$, particle size 10 $\mu \mathrm{m})$ with acetonitrile: water $(35: 65,20 \mathrm{ml} / \mathrm{min})$ was necessary. Detection was at $210 \mathrm{~nm}$ (KNAUER, "Smartline" UV detector 2500). The collected IS fraction from the HPLC was completely dried.

\subsection{LC-MS of the IS}

An Agilent 1100 HPLC system with UV-vis DAD detector and Agilent 6110 single-quadrupole MSD with electrospray ionisation were used. The column was a reversed phase C18 column (Grace Prevail RP-C18, $3 \mu \mathrm{m}$ particle, $150 \mathrm{~mm} \times 2 \mathrm{~mm}$ ). ESI was used in the negative mode.

The flow rate was $0.2 \mathrm{ml} / \mathrm{min}$. The following gradient was used: solvent $\mathrm{A}=$ water $+0.1 \%$ formic acid; solvent $\mathrm{B}=$ acetontrile. $0 \mathrm{~min}$ : $66 \% \mathrm{~A}, 34 \% \mathrm{~B} ; 2 \mathrm{~min}$ : $66 \%$ A, $34 \%$ B; 10 min: $58 \%$ A, $42 \%$ B; 16 min: $58 \%$ A, $42 \%$ B.

\subsection{HRMS of the IS}

The HRMS spectrum of the IS was recorded on a quadrupole orthogonal acceleration time-of-flight mass spectrometer (Synapt G2 HDMS, Waters, Milford, MA). The sample was infused at $3 \mu \mathrm{L} / \mathrm{min}$ and spectra were obtained in positive ionization mode with a resolution of 15,000 (FWHM) using leucine enkephalin as lock mass.

\subsection{NMR Analysis}

NMR spectra were recorded on a Bruker Avance 600 II+ spectrometer equipped with a $\mathrm{BBO}$ probe. The sample was dissolved in pyridine- $\mathrm{d}_{5}$ and chemical shifts were referenced to the solvent signal (for ${ }^{1} \mathrm{H}$ NMR the pyridine $\mathrm{H} 2$ signal was calibrated at $\delta=7.22 \mathrm{ppm}$; for ${ }^{13} \mathrm{C}$ NMR, the pyridine $\mathrm{C} 2$ signal was calibrated at $\delta=123.87 \mathrm{ppm})$.

\subsection{Analytical HPLC of Steviol Glycosides and IS}

All SVgly samples were analysed using analytical HPLC (Shimadzu Prominence) on two Grace Alltima $\mathrm{C}_{18}$ columns in series $(250 \mathrm{~mm} \times 4.6 \mathrm{~mm}$, particle size $5 \mu \mathrm{m}$ ) using an acetonitrile: $0.1 \% \mathrm{H}_{3} \mathrm{PO}_{4}$ gradient ( 0 - 2 min: $34 \% \mathrm{AcCN} ; 2$ - 10 min: $32 \% \rightarrow 42 \%$; 10 - 16 min: 42\%; $16.1 \mathrm{~min}: 34 \%)$. UV-detection was at $200 \mathrm{~nm}$ (Shimadzu, SPD-6A). The injection volume was $20 \mu \mathrm{L}$. 


\subsection{Preparation of Calibration Samples}

The following dried and very pure standards ( $>99 \%$ purity) were used to make calibration curves: RebA, ST, rebaudioside B (RebB) and steviolbioside (SB). The concentrations of RebA and ST varied between 0.032 and $0.517 \mathrm{mM}$ and these of RebB and SB between 0.0162 and $0.259 \mathrm{mM}$, respectively. The concentration of the IS was fixed at $0.125 \mathrm{mg} / \mathrm{mL}$ (ethanol:water $\mathrm{v} / \mathrm{v} ; 50: 50$ ) and all dilutions were made with this concentration of IS. Each calibration curve was made using 5 different concentrations of standards. As RebB and SB normally occur in much smaller amounts, the calibration curves were made with smaller concentrations for these 2 compounds. These 4 standards are good model compounds for the different SVglys: 2 of them are neutral (RebA, ST) and 2 contain a carboxylic function (RebB and SB). Previously, it has been shown that all SVglys have similar molar extinction coefficients [7] [10].

\subsection{Preparation of Samples for the Standard Addition Test: Accuracy Test}

The standard addition test gave an indication of the accuracy of analysis. Solutions of RebA were prepared and added to vials containing a fixed amount of IS. The first series of vials contained an "unknown amount" of RebA per vial (vials number 1). To these vials different amounts of RebA were added: 26.6, 51 and $71 \mu \mathrm{g}$, to vial numbers $2-4$, respectively. This allowed to measure the amount of RebA in the first series of vials, and also to calculate the amount after plotting the trend line through the peak ratios (RebA/IS) of the vials to which 3 known amounts of RebA were added. The intercept with the $y$-axis should be the same as the amount found for vial 1.

\subsection{Sample Clean-Up by SPE}

In order to test if the method could be used when sample-clean up is needed (e.g., analysis of food), we used a food matrix (Ice-Tea) and added different amounts of RebA $(0.075 \mathrm{mM})$ of ST $(0.094 \mathrm{mM})$. The Ice-Tea $(0.5 \mathrm{~mL})$ containing RebA $(0.075 \mathrm{mM})$ was spiked with $0.5 \mathrm{~mL}$ of 3 different RebA solutions (0.903 mM, $0.301 \mathrm{mM}$ or $0.1 \mathrm{mM}$ ). To this mixture $1 \mathrm{~mL}$ IS stock solution was added. Analogously, Ice-Tea $(0.5 \mathrm{~mL})$ containing ST $(0.094 \mathrm{mM})$ was spiked with ST (1.13 mM, $0.38 \mathrm{mM}$ or $0.13 \mathrm{mM})$. These samples were cleaned using the clean-up step. $\mathrm{C}_{18} \mathrm{SPE}$ columns of $0.5 \mathrm{~g}$ were conditioned with $\mathrm{MeOH}(3 \mathrm{~mL})$ followed by water $(3 \mathrm{~mL})$. After the application of the sample $(2 \mathrm{~mL})$, the columns were rinsed with $20 \%$ acetonitrile in water $(3 \mathrm{~mL})$ and the steviol glycosides were eluted with $80 \%$ acetonitrile in water $(5 \mathrm{~mL})$ and the eluate used for HPLC.

\section{Results and Discussion}

\subsection{Identification of the IS by LC-MS and NMR}

LC-MS and NMR data confirmed the identity of the IS as 
19- $O$ - $\beta$-D-galactopyranosyl-13- $O-\beta$-D-glucopyranosyl-steviol. The LC-MS data (Figure 2) contain clear signals in the ESI-spectrum (negative mode) for the molecular ion at $\mathrm{m} / z 641.3[\mathrm{M}-\mathrm{H}]^{-}$and a formic acid (FA) adduct at $\mathrm{m} / z 687.3$ $[\mathrm{M}+\mathrm{FA}-\mathrm{H}]^{-}$(FA was added to the eluent). HRMS of the IS $\left(\mathrm{C}_{32} \mathrm{H}_{50} \mathrm{O}_{13}\right)$ showed $\mathrm{m} / \mathrm{z}$ peaks at 643.3315 for $[\mathrm{M}+\mathrm{H}]^{+}$(calculated value 643.33238), $660.3587\left[\mathrm{M}+\mathrm{NH}_{4}\right]^{+}$(calculated value 660.35893$)$ and $665.3140[\mathrm{M}+\mathrm{Na}]^{+}$ (calculated value 665.31436) (Figure 3).

NMR gave the following results:

${ }^{1} \mathrm{H}$ NMR (Pyridine- $\left.\mathrm{d}_{5}, 600 \mathrm{MHz}\right) \delta 6.10(1 \mathrm{H}, \mathrm{d}, J=7.8 \mathrm{~Hz}, \mathrm{H1}$ ”), $5.54(1 \mathrm{H}, \mathrm{br}$ s, H17), 5.14 (1 H, d, $J=7.5 \mathrm{~Hz}, \mathrm{H1}$ '), 4.99 (1 H, br s, H17), 4.67 - 4.58 ( $3 \mathrm{H}, \mathrm{m}$, $\mathrm{H} 2 "+\mathrm{H} 4 "+\mathrm{H} 6$ '), $4.47(1 \mathrm{H}, \mathrm{dd}, J=9.9,6.6 \mathrm{~Hz}, \mathrm{H} 6 "), 4.38(1 \mathrm{H}, \mathrm{dd}, J=10.3$, $\left.5.6 \mathrm{~Hz}, \mathrm{H6} 6^{\prime \prime}\right), 4.29$ (1 H, dd, J= 10.8, 6.4 Hz, H6'), $4.24-4.05$ (5 H, m, H2' + H3' + H4' + H3" + H5"), 3.97 (1 H, br s, H5'), 2.70 (1 H, d, J=11.3 Hz, H14), 2.47 $2.40(1 \mathrm{H}, \mathrm{m}), 2.36(1 \mathrm{H}, \mathrm{d}, J=13.2 \mathrm{~Hz}), 2.35-2.27(1 \mathrm{H}, \mathrm{m}), 2.26-2.17(1 \mathrm{H}$, m), $2.06(2 \mathrm{H}, \mathrm{s}, \mathrm{H} 15), 1.90-1.60(6 \mathrm{H}, \mathrm{m}), 1.43(1 \mathrm{H}, \mathrm{d}, J=15.3 \mathrm{~Hz}), 1.30-1.23$ $(8 \mathrm{H}, \mathrm{m}), 1.06-0.97(2 \mathrm{H}, \mathrm{m}), 0.90(1 \mathrm{H}, \mathrm{d}, J=7.1 \mathrm{~Hz}), 0.77(1 \mathrm{H}, \mathrm{t}, J=11.5 \mathrm{~Hz})$.

${ }^{13} \mathrm{C}$ APT NMR (Pyridine- $\left.\mathrm{d}_{5}, 151 \mathrm{MHz}\right) \delta 177.4$ (C19), 154.9 (C16), 104.8 (C17), 100.1 (anomeric), 96.7 (anomeric), $86.3(\mathrm{C} 13), 79.2(\mathrm{CH}), 78.4(\mathrm{CH}), 78.2(\mathrm{CH})$, $76.3(\mathrm{CH}), 75.9(\mathrm{CH}), 72.7(\mathrm{CH}), 71.7(\mathrm{CH}), 70.4(\mathrm{CH}), 63.4\left(\mathrm{C6}^{\prime}\right), 62.2(\mathrm{C6}$ ”),
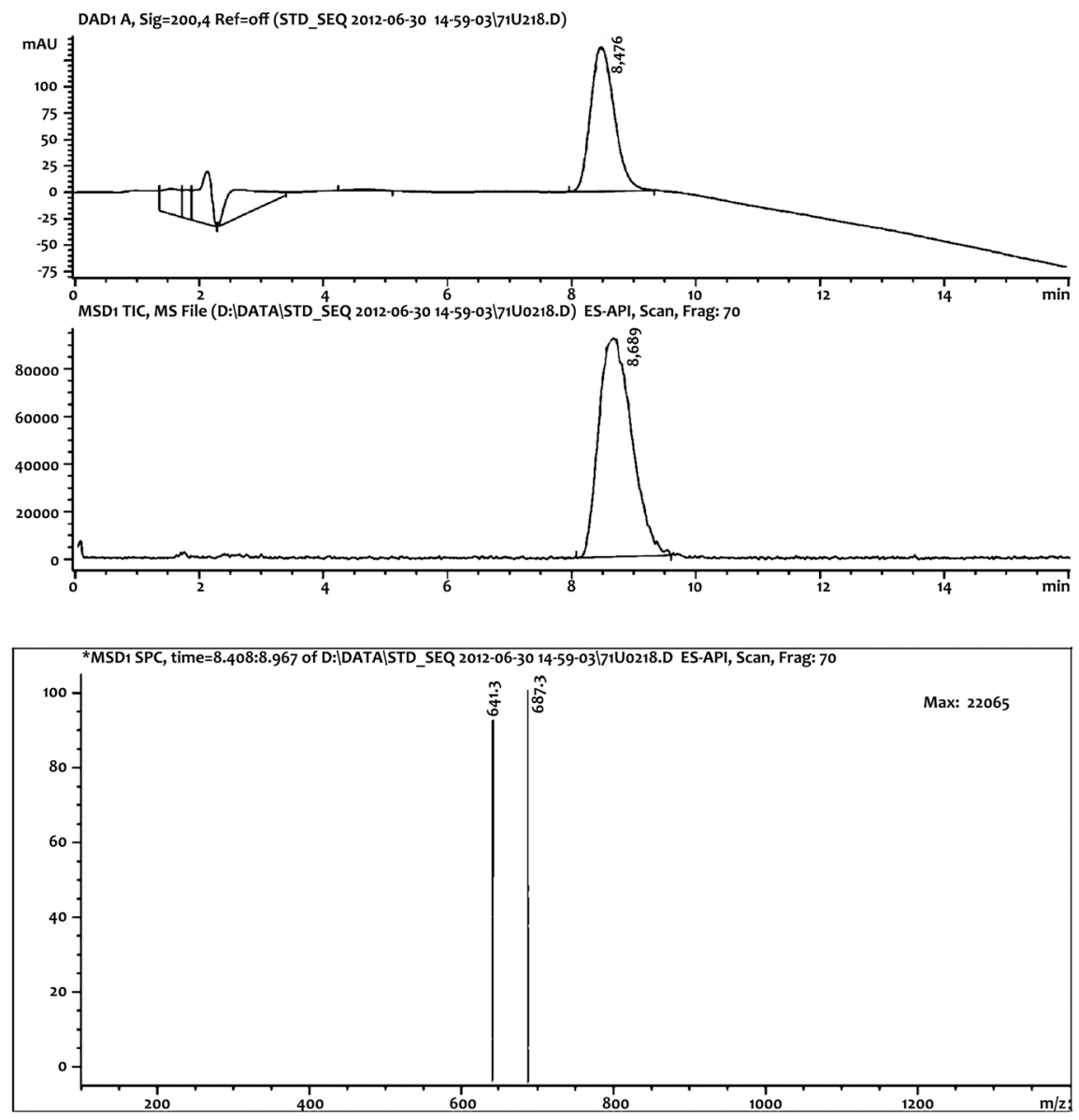

Figure 2. LC-MS data for the internal standard. 


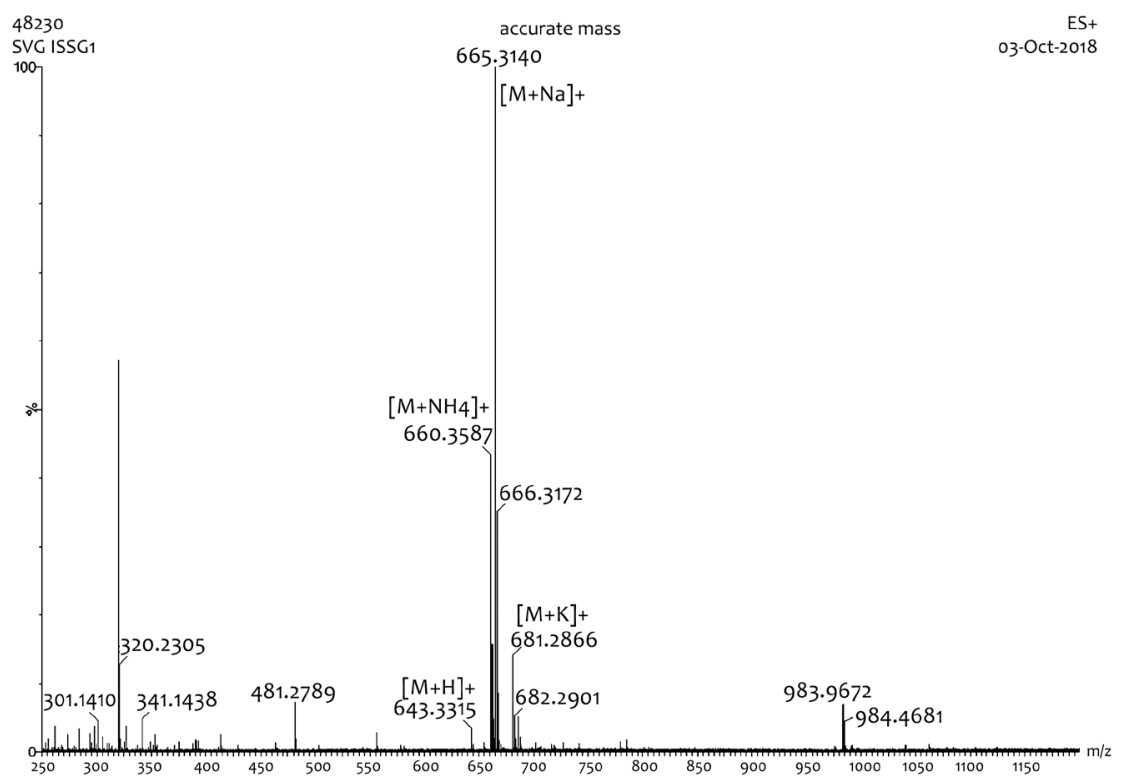

Figure 3. HRMS of the IS.

57.7 (C5), 54.3 (C9), 48.1 (C15), 44.9 (C14), 44.4 (C4), 42.8 (C8), 42.0 (C7), 41.1 (C1), 40.2 (C10), 38.7 (C3), 37.7 (C12), 28.8 (C18), 22.5 (C6), 21.0 (C11), 19.8 (C2), 16.0 (C20).

The ${ }^{13} \mathrm{C}$-NMR spectrum recorded in pyridine is in agreement with available literature data for the proposed structure with typical signals appearing in the ${ }^{13} \mathrm{C}$ NMR spectrum in pyridine- $\mathrm{d}_{5}$ at 104.8 (C17) and $154.9(\mathrm{C} 16)$ for the steviol alkene moiety and at 96.7 and 100.1 for the anomeric carbon atoms of the sugar residues ( $\mathrm{C} 1$ of $19-O-\beta$-Gal and $\mathrm{C} 1$ of $13-O-\beta$-Glc, respectively) [17].

\subsection{Use of the IS}

First of all, it had to be proved that the IS was well separated from the other SVglys and that there were no interfering components in the mixture without the IS added. Figure 4 shows the HPLC analysis of a sample of IS (A), a commercial mixture of SVgly (C) and of a co-injection of both samples (B). It is clear that the sample without IS doesn't contain interfering peaks at the expected position of the IS and that the IS is very well separated from Rub.

\subsection{Sample Clean-Up}

The IS is intended not only for measuring mixtures of purified SVglys, but also for crude plant extracts. Then it should be possible to purify crude extracts by SPE. Of course, in the SPE step the IS should behave as the SVglys. Therefore, it is of utmost importance that the peak ratios are the same before and after SPE. To test this, $1 \mathrm{~mL}$ of IS solution was added to $1 \mathrm{~mL}$ RebA or ST solution. Three different RebA and ST concentrations were used. HPLC analysis of the mixtures was done before and after the clean-up step. The peak ratios between RebA or ST and the IS were calculated and plotted against the RebA or ST concentration (Figure 5). 


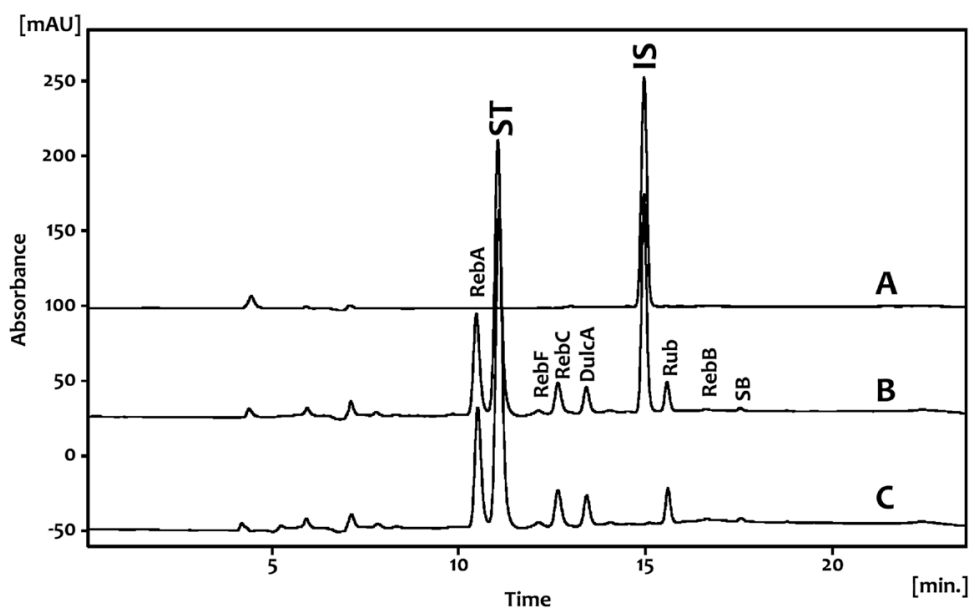

Figure 4. HPLC trace of (A) IS; (B) co-injection of IS with a commercial SVgly mixture and $(\mathrm{C})$ commercial SVgly mixture.

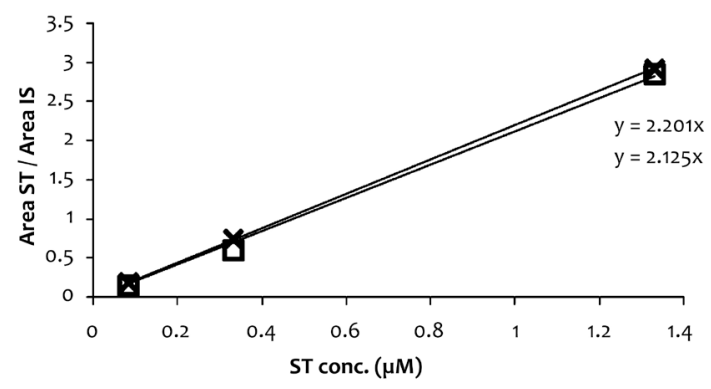

(a)

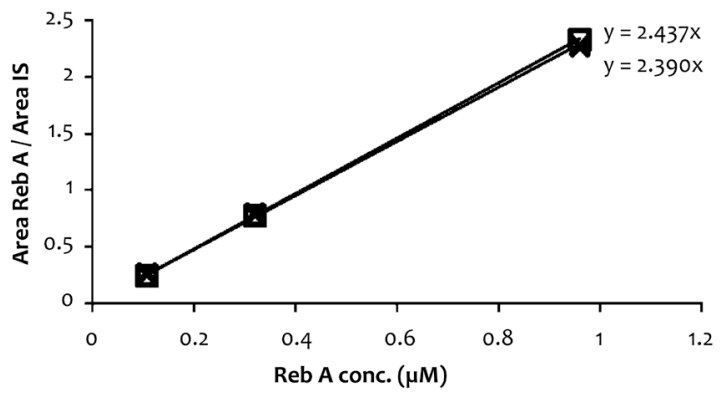

(b)

Figure 5. (a) Area ST/Area IS plotted against the used ST concentration before $(X)$ and after ( $\square$ ) sample clean-up; (b) Area RebA/Area IS plotted against the used RebA concentration before $(\times)$ and after $(\square)$ sample clean-up.

Figure 5 shows that the peak ratios of the SVgly over IS were constant before and after the SPE purification step, proving that there was no problem in using a purification step in the quantification of SVgly.

\subsection{Calibration Curves}

An example of the separation of the standards and the IS is given in Figure 6. The fluctuation in the baseline between 5.1 and $7.5 \mathrm{~min}$ is due to the ethanol present in the solvent. 


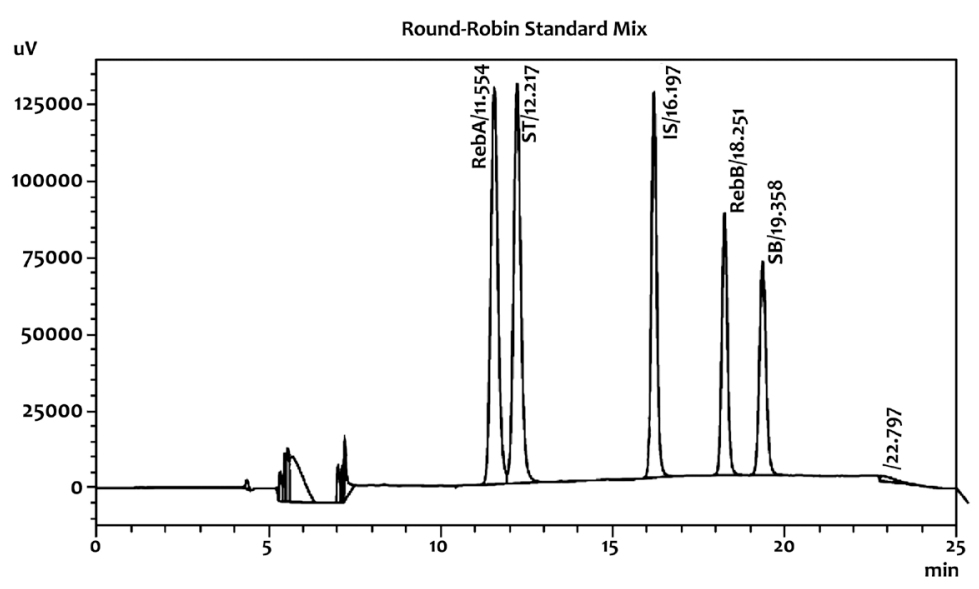

Figure 6. Order of elution of the different standards and the internal standard (RebA: $\pm 11.5 \mathrm{~min}$; stevioside: $\pm 12.2 \mathrm{~min}$; IS: $\pm 16.2 \mathrm{~min}$; RebB: $\pm 18.2 \mathrm{~min}$; $\mathrm{SB}: \pm 19.5 \mathrm{~min}$ ).

The calibration curves were linear and the trend lines could be forced through zero giving a perfect linear function $(y=m \times x)$ with $\mathrm{m}$ (and $\left.\mathrm{R}^{2}\right)$ values of 5.376 (0.9999), 5.3947 (0.9998), 5.4646 (1), and 5.3677 (1), for RebA, ST, Reb B and SB, respectively.

There is almost no difference between these 4 equations, as the steviol glycoside concentrations are plotted in function of their $\mathrm{mM}$ concentration. It has been shown earlier that the extinction coefficients of all SVgly are very similar, hence very similar calibration curves can be expected [7] [10].

\subsection{LOD and LLOQ}

The molar extinction coefficients of the different SVglys were measured in different solvents. As the HPLC solvents are mixtures of AcCN/water, the average ( \pm se) extinction coefficients were 6214 and 7697 in $80 \%$ and $35 \%$ AcCN, respectively [11].

The limit of detection (LOD) by an UV detector at $210 \mathrm{~nm}$ is about $25 \mathrm{ng}$ (Knauer Smartline UV detector 2500:noise ratio 5:1), at $197 \mathrm{~nm}$ about $10 \mathrm{ng}$.

The lower limit of quantification (LLOQ) is $125 \mathrm{ng}$ at $210 \mathrm{~nm}$, and $50 \mathrm{ng}$ at $197 \mathrm{~nm}$ (signal/noise ratio: 5/1).

The range of measurements for steviol glycosides is between 0.25 and $1000 \mu \mathrm{g} / \mathrm{mL}$ (or between about 15 and $1250 \mu \mathrm{M}$ ).

\subsection{Precision of the Method}

The inter-laboratory precision of the method can be deduced from the percentages RSD of 3 injections of the mixture with standards (Table 1). The values were obtained from the ratios of standards over IS. By injection of the standards, most of the laboratories could reproduce the calibrations with an RSD below $0.5 \%$, and a few labs arrived at very small values under $0.1 \%$. Further results of round-robin testings with an IS will be given in a next publication.

Using the standard addition method, the accuracy of the method could be evaluated (Figure 7). The theoretical RebA amount in vials 1 was $50.4 \mu \mathrm{g}$. The 


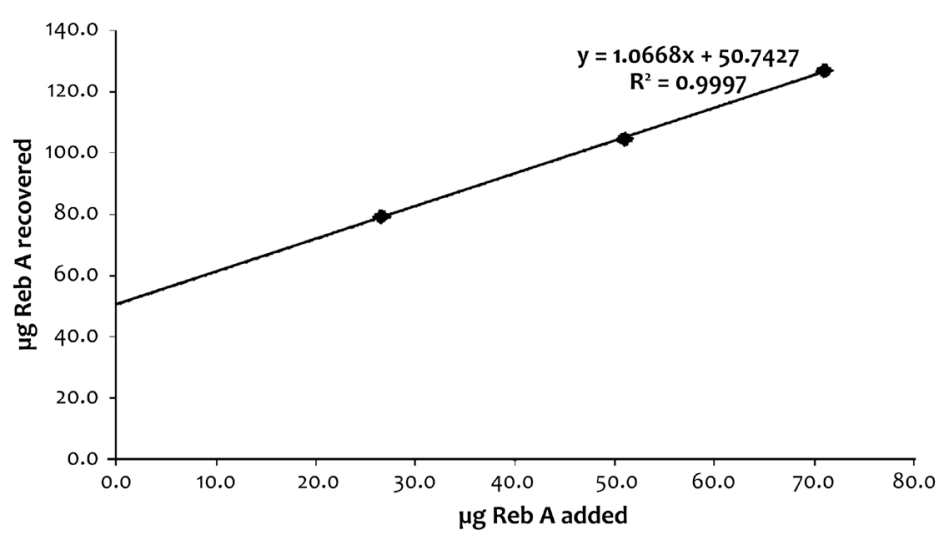

Figure 7. Standard addition curve for RebA.

Table 1. Percentages RSD for 4 compounds after 3 injections of the calibration mixture. Ten laboratories performed the analyses.

\begin{tabular}{ccccc}
\hline Lab \# & RebA & ST & RebB & SB \\
\hline 1 & 0.126 & 0.138 & 0.236 & 0.232 \\
5 & 0.238 & 0.158 & 0.344 & 0.423 \\
7 & 0.186 & 0.794 & 0.678 & 1.551 \\
19 & 0.710 & 0.741 & 0.123 & 0.256 \\
24 & 0.063 & 0.082 & 0.027 & 0.070 \\
27 & 0.790 & 0.744 & 0.671 & 1.134 \\
28 & 0.079 & 0.046 & 0.135 & 0.135 \\
30 & 0.196 & 0.099 & 0.548 & 0.180 \\
31 & 0.254 & 0.412 & 0.130 & 0.121 \\
32 & 0.010 & 0.010 & 0.012 & 0.015 \\
\hline
\end{tabular}

Accuracy: Standard addition test.

calculated average of the RebA amount obtained by the intercept of the trend line was $50.7 \mu \mathrm{g}$. This is $100.59 \%$ of the theoretical value, showing the accuracy of the analysis.

\section{Analysis of a Sample of SVglys with the IS Method}

One gram of a SVgly solution $(1.5 \mathrm{mg} / \mathrm{g})$ was added to a vial containing IS. The chromatogram is shown in Figure 8.

Table 2 gives the amounts of the different SVglys ( $\mathrm{mg} / \mathrm{g}$ solution) as well as the purity of the unknown sample of Figure 8 (purity: 96.6\%). More details of inter-laboratory analysis by the IS method will be given in an upcoming publication.

\section{Conclusion}

An IS could be synthesised that behaved like SVglys in all the steps of handling and is well separated from the known SVglys. The LOD and LLOQ were sufficient 


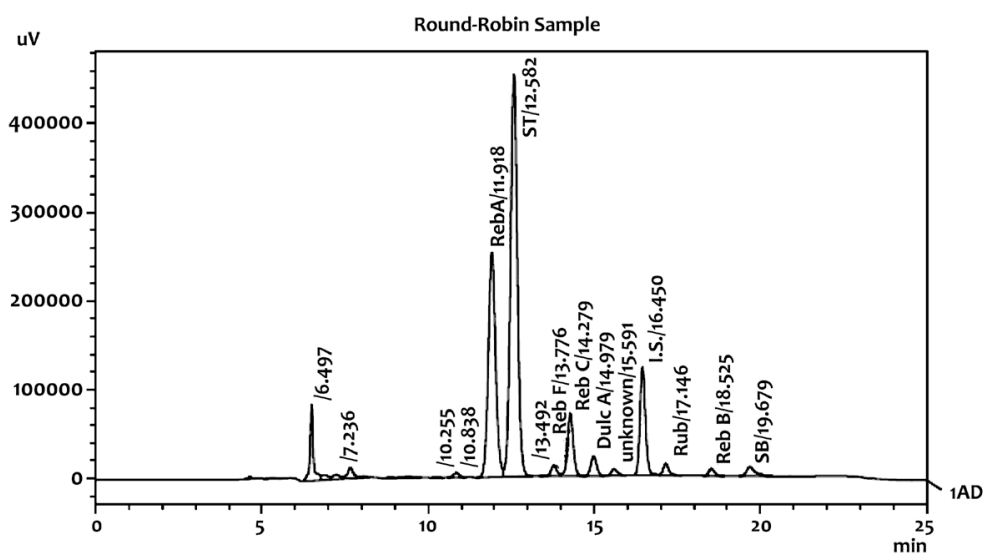

Figure 8. Example of the analysis of a commercial sample after the addition of IS. RebD: 7.236; RebE: 10.838; RebA: 11.918 min; stevioside: 12.582; RebF: 13.776; RebC: 14.279; DulA: 14.979; unknown: 15.991; IS: 16.450; rubusoside: 17.146; RebB: 18.525; steviolbioside: 19.679 .

Table 2. Quantitative analysis of an unknown sample. The values are corrected for different molecular masses and for water content of the unknown sample. Values are given in $\mathrm{mg} / \mathrm{g}$ solution.

\begin{tabular}{cccccccccccc}
\hline Lab \# & RebD & RebE & RebA & ST & RebF & RebC & DulA & Rub & RebB & SB & Purity \\
\hline 6 & 0.016 & 0.0094 & 0.489 & 0.679 & 0.0183 & 0.096 & 0.0288 & 0.0138 & 0.011 & 0.0168 & $96.6 \%$ \\
\hline
\end{tabular}

as the availability of sample mass is not a problem. A base-line separation of RebA and ST is required for good peak integration. The inter-laboratory RSD for the calibration curves for most laboratories was $<0.5 \%$ and a few laboratories obtained a value $<0.1 \%$. The deviation in the accuracy test was below $0.6 \%$. An analysis of a commercial sample could be done without showing any problems. In a next publication, the results will be shown of round-robin testings organised by EUSTAS (European Stevia Association) to see if an inter-laboratory RSD around $1 \%$ would be possible.

\section{Acknowledgements}

The authors acknowledge the financial support from Medherbs, Wiesbaden, Germany, and Stepaja, Aarschot, Belgium. None of the funding organisations had any role in the design and conduct of the study; collection, management, analysis, and interpretation of the data; and/or preparation, review, or approval of the manuscript. Mass spectrometry was made possible by the support of the Hercules Foundation of the Flemish Government (Grant 20100225-7). The Hercules Foundation is acknowledged for supporting the purchase of a $400 \mathrm{MHz}$ NMR spectrometer through project AKUL1311.

\section{Conflicts of Interest}

The authors declare no conflicts of interest regarding the publication of this paper. 


\section{References}

[1] FSANZ (2008) Final Assessment Report, Application A540, Steviol Glycosides as Intense Sweeteners, 100.

[2] JECFA (2008) Steviol Glycosides. FAO JECFA Monograph 5.

[3] JECFA (2010) Steviol Glycosides. FAO JECFA Monograph 10.

[4] EFSA (2010) Scientific Opinion on the Safety of Steviol Glycosides for the Proposed Uses as a Food Additive. EFSA Journal, 8, 1537

[5] EU (2011) Commission Regulation (EU) No. 1131/2011. Official Journal of the European Union, L295, 205-211.

[6] Scaglianti, M.C. Gardana, P.G. Pietta and Ricchiuto, G.M. (2008) Analysis of the Main Stevia rebaudiana Sweeteners and Their Aglycone Steviol by a Validated LC-DAD-ESI-MS Method. Proceedings of the EUSTAS Stevia Symposium, KU Leuven, 27 June 2008, 45-58.

[7] Geuns, J.M.C. (2010) Stevia and Steviol Glycosides. Euprint Editions, Heverlee, 307.

[8] Gardana, C., Scaglianti, M. and Simonetti, P. (2010) Evaluation of Steviol and Its Glycosides in Stevia rebaudiana Leaves and Commercial Sweetener by Ultra-High-Performance Liquid Chromatography-Mass Spectrometry. Journal of Chromatography A, 1217, 1463-1470. https://doi.org/10.1016/j.chroma.2009.12.036

[9] Geuns, J.M.C. (2018) Inter-Laboratory Analysis of Steviol Glycosides by an external Standard Method (Submitted).

[10] Geuns, J.M.C. and Struyf, T. (2009) EUSTAS Round-Robin Testing of Steviol Glycosides Proceedings of the 3rd EUSTAS Stevia Symposium "Stevia in Europe", KU Leuven, 1-2 July 2009, 35-48.

[11] Geuns J.M.C. (2008) Analysis of Steviol Glycosides: Validation of the Methods. Proceedings of the EUSTAS Stevia Symposium, KU Leuven, 27 June 2008, 59-78

[12] Molina-Calle, M., Sánchez de Medina, V., Delgado de la Torre, M.P., Priego-Capote, F. and Luque de Castro, M.D. (2016) Development and Application of a Quantitative Method Based on LC-QqQ MS/MS for Determination of Steviol Glycosides in Stevia Leaves. Talanta, 154, 263-269. https://doi.org/10.1016/j.talanta.2016.03.051

[13] Zimmermann, B.F. and Wölwer-Rieck, U. (2011) HILIC Columns for the Analysis of Steviol Glycosides. Proceedings of the 5th EUSTAS Stevia Symposium Stevia, Breakthrough in Europe, KU Leuven, 28-29 June 2011, 147-152.

[14] Stijn, C. and Geuns, J.M.C. (2013) Steviol Glycosides: Chemical Diversity, Metabolism and Function. Journal of Natural Products, 76, 1201-1228. https://doi.org/10.1021/np400203b

[15] Struyf, T., Chandia, N.P., De Borggraeve, W., Dehaen, W. and Geuns, J.M.C. (2008) Preparation of Pure Standards of Steviol Glycosides. Identification of Steviol Glycosides by LC-MS and NMR. Proceedings of the EUSTAS Stevia Symposium, KU Leuven, 27 June 2008, 29-44.

[16] Ogawa, T., Nozaki, M. and Matsui, M. (1980) Total Synthesis of Stevioside. Tetrahedron, 36, 2641-2648. https://doi.org/10.1016/0040-4020(80)80136-0

[17] Mizutani, K., Miyata, T., Kasai, R., Tanaka, O., Ogawa, S. and Doi, S. (1989) Study on Improvement of Sweetness of Steviol Bisglycosides: Selective Enzymic Transglucosylation of the 13-O-Glycosyl Moiety. Agricultural and Biological Chemistry, 53, 395-398. 


\section{Abbreviations}

Using SV for steviol, allows the use of the following abbreviations: SVgly(s): steviol glycoside(s); SM: steviolmonoside; ST: stevioside; RebA-G: rebaudioside A-G; SB: steviolbioside; DulA: dulcoside A; Rub: rubusoside. 